\title{
Tendenze recenti della crescita insediativa. Il contributo della banca dati Uso e Copertura del Suolo di Regione Toscana (2007/2010/2013)
}

\author{
Christian Ciampi $\left({ }^{a}\right)$, Lorenzo Bottai $\left({ }^{b}\right)$, Bruno Giusti $\left({ }^{a}\right)$, Manuela Corongiu $\left({ }^{b}\right)$, Fabio Lucchesi $\left({ }^{a}\right)$ \& Umberto Sassoli $\left({ }^{c}\right)$
}

$\left({ }^{a}\right)$ Dipartimento di Architettura, Laboratorio di Cartografia, Università di Firenze, Via della Mattonaia 14, Firenze. E-mail: christian.ciampi@gmail.com
(b) LaMMA, Laboratorio di Monitoraggio e Modellistica Ambientale per lo sviluppo sostenibile.

(c) Regione Toscana, Sistema Informativo Territoriale e Ambientale.

\section{ABSTRACT}

Il contributo intende presentare i risultati di alcune valutazioni sulle transizioni degli usi del suolo intervenute nel territorio toscano nel periodo 2007/2013, con particolare attenzione alla progressiva sottrazione di suolo agricolo per l'espansione delle aree artificiali. La natura del fenomeno indagato, spesso indicata nel dibattito con l'espressione "consumo di suolo", rende questo contributo potenzialmente importante dal punto di vista metodologico e riproducibile in altri contesti e a diverse scale (EEA, 2006b). Tali valutazioni sono rese possibili dal programma di aggiornamento triennale del tematismo Uso e Copertura del Suolo (UCS) in scala nominale $1 / 10.000$, e in coerenza con la cartografia tecnica regionale (CTR) 1/10.000, promossa da Regione Toscana dal 2007.L'analisi delle diverse categorie di transizione (svolta attraverso il modello Land Cover Flows, EEA 2001) non è basata esclusivamente su criteri quantitativi; l'indagine, infatti, tenta una valutazione della distribuzione spaziale dei fenomeni descritti. In primo luogo i cambiamenti sono messi in relazione con diverse articolazioni del territorio regionale identificate attraverso classificazioni paesaggistiche, storiche, amministrative. In secondo luogo viene sperimentato l'utilizzo dei metodi di misura delle metriche spaziali tipiche della landscape ecology; tali metodi consentono di definire, pur attraverso parametri quantitativi, i caratteri localmente definiti delle trasformazioni. Scopo della ricerca, in sintesi, è l'interpretazione e la rappresentazione delle dinamiche del cambiamento attraverso la loro caratterizzazione locale. Ogni trasformazione è intrinsecamente legata alle caratteristiche del territorio in cui trova spazio, dunque non solo dall'insieme degli elementi fisici e storici che lo caratterizzano, ma anche dai processi di gestione e pianificazione che ne indirizzano il potenziale cambiamento attraverso indirizzi e azioni di pianificazione.

KEY WORDS: consumo di suolo, impegno di suolo, pressione insediativa, Corine Land Cover (CLC), Land Cover Flow (LCF).

\section{IL MODELLO LAND COVER FLOW PER L'INTERPRETAZIONE DELLA CRESCITA INSEDIATIVA}

Nel corso degli ultimi anni la Regione Toscana si è dotata di un programma di acquisizione e di aggiornamento del tematismo Uso e Copertura del Suolo (UCS) realizzato, per tutto il territorio regionale, a partire dall'analisi delle foto aeree digitali acquisite con intervallo temporale triennale. Al momento la banca dati UCS è stata realizzata e aggiornata con le riprese aeree del 2007, 2010 e 2013 (Trevisani \& Sassoli, 2014).

La banca dati UCS può essere utilmente esplorata attraverso l'uso del modello Land Cover Flows (LCF) (EEA, 2001), che classifica, sulla base di specifiche significatività, le combinazioni possibili nelle transizioni tra le categorie previste dal terzo livello della legenda Corine Land Cover (CLC) (Bossard et al., 2000) (EEA, 2006a). Tra le classi previste dal modello LCF alcune esprimono direzioni di cambiamento particolarmente significative nella valutazione dell'intensità del consumo di suolo. In particolare si può fare riferimento: (1) alla transizione da qualsiasi classe di superfici non artificiali alle classi delle aree residenziali, che è raccolta in un'unica voce LCF, denominata "lcf 2: Urban residential sprawl"; (2) alla transizione da qualsiasi classe di superfici non artificiali alle classi delle aree produttive e commerciali, che è raccolta nella voce denominata "lcf 3: Sprawl of economic sites and infrastructures".

Nell'intervallo 2007/2013, malgrado la crisi economica, 7.162 ettari di territorio regionale (lcf $2+\operatorname{lcf} 3$ ) sono stati destinati a nuova edificazione, per lo più in funzione di aree produttive e per infrastrutture.

Indagando ad un livello di disaggregazione più fine, si segnalano i valori delle categorie lcf 22 (Urban diffuse residential sprawl) e lcf 31 (Sprawl of industrial \& commercial sites), che sono le classi più rappresentate. La progressiva sottrazione di suolo per l'espansione degli insediamenti dunque sembra ancora intensa nel periodo 2007/2013.

\section{DISTIBUZIONE SPAZIALE DELLA CRESCITA INSEDIATIVA}

La risoluzione spaziale natura spaziale della banca dati UCS permette di verificare, in maniera ottimale, se la tendenza è costante in tutte le parti del territorio regionale, oppure, per esempio, se le diverse condizioni fisiografiche la rendono più $\mathrm{o}$ 
meno potente, ed eventualmente, con quali diverse intensità. Per valutare se questo fenomeno è costante in tutte le parti del territorio regionale sono state utilizzate alcune subregioni caratterizzate da una qualche omogeneità fisiografica (Rossi et al., 1994), che, semplificando,potremmo associare alla manifestazione di una peculiare identità paesaggistica. Tali subregioni sono un'articolazione dei paesaggi toscani fondata sulla caratterizzazione geomorfologica, idrografica, climatica e vegetazionale definita da Aldo Sestini (Sestini, 1963). Questa articolazione propone per la Toscana nove sistemi di paesaggio: Rilievi dell'Antiappennino, Ripiani Tufacei, Appennino, Colline Plioceniche, Alpi Apuane, Isole e Promontori, Conche Intermontane, Pianura Costiera, Pianura Alluvionale.

\section{INDICATORI DEL CONSUMO DI SUOLO}

Lo studio, con lo scopo di evidenziare i caratteri del fenomeno del consumo di suolo in prospettive temporali diverse, in particolare distinguendo il lungo periodo dell'evoluzione insediativa dalle tendenze rilevabili nella contemporaneità, propone una serie differenziata di indicatori.

Un primo indicatore, l'indice di impegno di suolo (IS), misura la diversa incidenza delle superfici artificializzate rispetto alla superficie territoriale, dando conto del fenomeno nella sua lunga durata:

\section{(superficie artificiale 2013 / superficie territoriale) * 100}

Un secondo indicatore, l'indice di consumo di suolo (CS), definisce il rapporto tra quantità delle superfici artificializzate in un dato intervallo di tempo $\left(\mathrm{T}_{1}-\mathrm{T}_{0}\right)$ e superficie artificiale alla prima soglia temporale considerata $\left(\mathrm{T}_{0}\right)$ :

\section{(superficie artificiale 2013 - superficie artificiale 2007)/ (superficie artificiale 2007) *100}

Il confronto tra la distribuzione spaziale di questi due indicatori mostra come non ci sia un rapporto di diretta

proporzionalità tra impegno e consumo di suolo: se tale rapporto ci fosse, le superfici artificializzate, ambito fisiografico per ambito fisiografico, dovrebbero crescere in quantità commisurate all'estensione delle superfici artificiali esistenti all'inizio del periodo (come farebbe il capitale finanziario). Poiché questa perfetta relazione di proporzionalità non è rilevata, come leggibile in Figura 1, allora esistono evidentemente altri fattori influenti, legati ai caratteri delle diverse regioni toscane, o, per meglio dire, al modo in cui il mercato apprezza, momento per momento le conseguenze dell'artificializzazione delle diverse regioni toscane.

Il grafico mostra che sono le pianure alluvionali (Piana Fiorentina, Medio Valdarno e Valdarno Inferiore, Valdinievole, Piana di Lucca) a conoscere le maggiori intensità di consumo di suolo; ma evidenzia anche che il periodo $2007 / 2013$ ha presentato la tendenza di altre aree a crescere

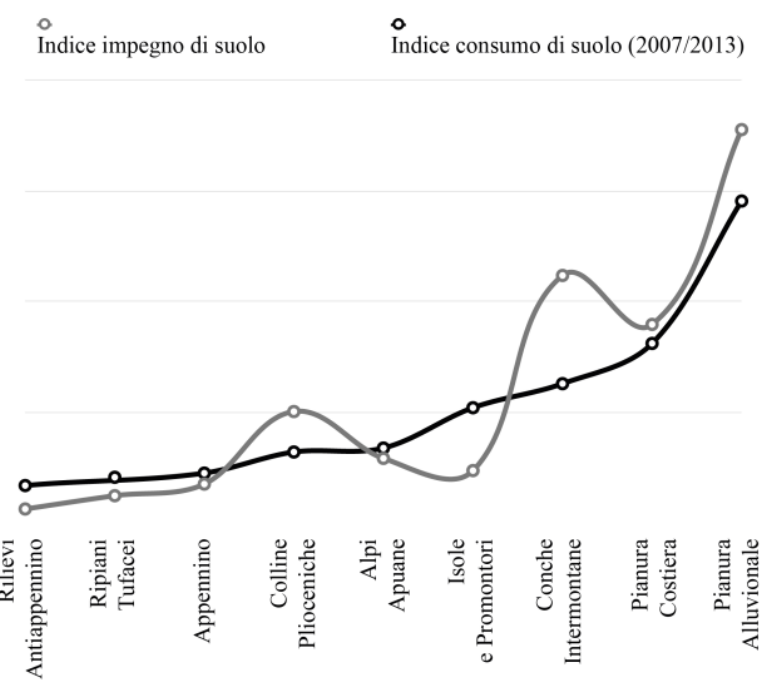

Fig. 1 - Visualizza i rapporti tra i valori dell'indice CS e del'indice IS.

oltre le misure definite dalla propria dotazione iniziale: le conche intermontane sub metropolitane (essenzialmente: Mugello, Valdarno Superiore) e i paesaggi celebrati delle colline plioceniche (essenzialmente: Val di Pesa, Valdelsa e Chianti).

\section{L'INDICE DI PRESSIONE INSEDIATIVA. L'ESEMPIO DELLA COSTA TOSCANA}

I due indici individuati possono essere messi in relazione tra di loro per mostrare, come in un piccolo intervallo di tempo, si possano manifestare particolari pressioni insediative.

A tal proposito si introduce un nuovo indicatore, l'indice $d i$ pressione insediativa (IP), dato dal rapporto tra $\mathrm{i}$ due indici presentati sopra:

\section{$C S / I S$}

Per verificare l'incidenza delle politiche urbanistiche alla scala locale lo studio ha isolato un ambito fisiografico omogeneo: la costa toscana. I valori dei tre indici sono stati calcolati per l'estensione di ciascun dei 35 comune compresi in questo ambito.

L'analisi dei dati mostra come, in contesti specifici, una pressione insediativa molto rilevante possa nascondersi a valori di CS molto bassi. In questi casi è IP che esplicita le tendenze in atto (Fig. 2) .Si noti in particolare i casi di Castagneto Carducci e Magliano in Toscana, che pur presentando indici CS tra i più bassi della costa, fanno registrare IP molto alti. Viceversa Forte dei Marmi, a fronte di un IS elevato, riporta un IP che risulta essere tra $\mathrm{i}$ più bassi. Si tratta di due casi esemplari dei processi di artificializzazione dei suoli in atto in questo contesto: la crescente domanda immobiliare in ambiti turistici non ancora saturi (Castagneto Carducci, Magliano in Toscana) e la saturazione dei contesti quasi completamente urbanizzati (Forte dei Marmi) significativi di nuova artificializzazione 

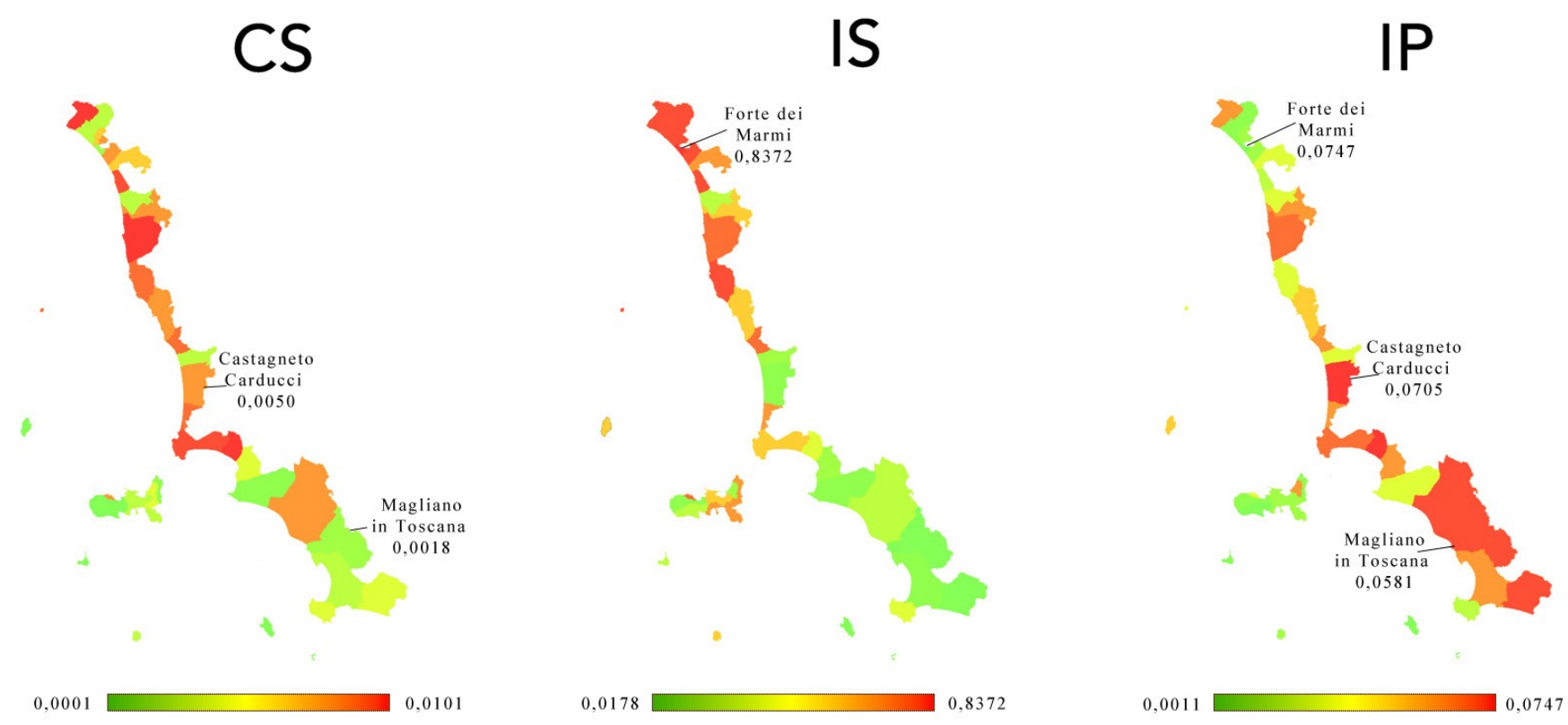

Fig. 2 - Gli indici dei comuni della costa toscana.

\section{CONCLUSIONI E PROSPETTIVE DI RICERCA}

L'uso degli indici quali quelli utilizzati in questo contesto aggiunge uno strumento efficace per la corretta interpretazione delle tendenze evolutive del fenomeno del consumo di suolo. In particolare, l'affiancamento di diverse articolazioni spaziali per la valutazione di questo fenomeno (secondo criteri fisiografici e, insieme, secondo criteri amministrativi) ne migliora la capacità di comprensione, soprattutto alla luce di quelle che sono le politiche di gestione dei processi di pianificazione alla scala regionale, ma anche comunale.

In questa direzione di ricerca un possibile strumento di approfondimento è lo studio delle diverse forme delle espansioni insediative (Cusmano, 1996).

Le prime riflessioni che vogliamo condividere sono relative a due indicatori di forma MPS (Mean Patch Size) e MedPS (Median Patch Size) (McGarigal \& Marks, 1995; McGarigal et al., 2012) che valutano le aree di nuova artificializzazione in base alla loro dimensione media e mediana.

Soprattutto il dato della mediana delle superfici artificializzazione intervenuti nell'intervallo 2007-2013, può evidenziare una diversa gestione dei processi di pianificazione urbana e territoriale (Fig.3).

Più l'indicatore di forma MedPS ha valori bassi, più la dimensione delle patches relativa agli interventi di nuova artificializzazione (il $50 \%$ sul totale) avrà una dimensione contenuta. Viceversa a valori alti dell'indicatore corrisponderanno dimensioni più grandi delle patches di nuova artificializzazione.

In sintesi, è possibile constatare come, interventi dalle dimensioni contenute siano tipicamente riferibili all'utilizzo dell'istituto dell'intervento diretto, mentre aree di
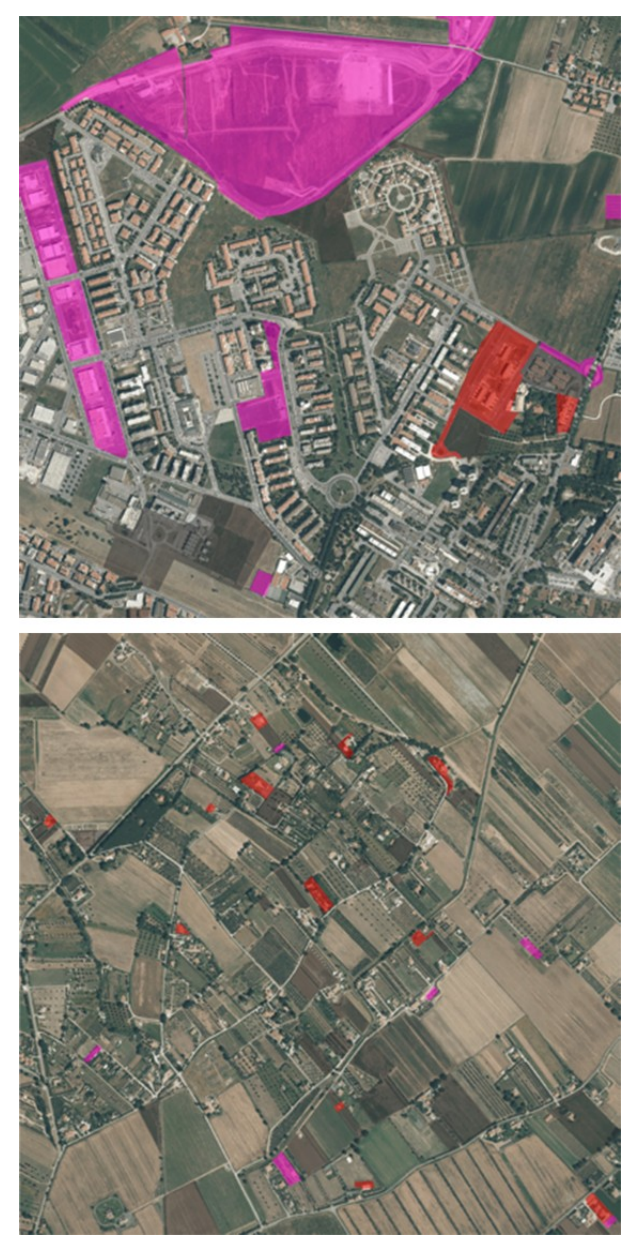

Fig. 3 - In blu esempi di processi di pianificazione attuativa, in rosso esempi di intervento diretto. 
trasformazione di grandi dimensioni siano il tipico esito di processi di pianificazione attuativa.

Questo ulteriore criterio di valutazione restituisce alle attività istituzionali di governo del territorio l'effettivo ruolo nella determinazione del fenomeno del consumo di suolo.

\section{RIFERIMENTI BIBLIOGRAFICI}

Bossard M., Feranec J. \& Otahel J. (2000) - CORINE Land Cover Technical Guidelines - Addendum 2000, European Environment Agency, 105 pp.

Cusmano M.G. (1996) - Misura misurabile. Argomenti intorno alla dimensione urbana. Franco Angeli, Milano, 112 pp.

EEA (2001) - Changes Classification: Land Cover Flows, European Environment Agency, $10 \mathrm{pp}$.

EEA (2006a) - CORINE Land Cover, Technical Guideline, European Environment Agency, $70 \mathrm{pp}$.

EEA (2006b) - Urban sprawl in Europe. The ignored challenge,
European Environment Agency, 60 pp.

McGarigal K. \& Marks B.J. (1995) - FRAGSTAT: Spatial Pattern Analysis Program for quantifying landscape structure, Gen. Tech. Rep. PNW-GTR-351. Portland, OR: U.S. Department of Agriculture, Forest Service, Pacific Northwest Research Station, 122 pp.

McGarigal K., Cushman S.A. \& Ene E. (2012) - FRAGSTATS v4: Spatial Pattern Analysis Program for Categorical and Continuous Maps, Computer software program produced by the authors at the University of Massachusetts, Amherst, $182 \mathrm{pp}$.

Rossi R., Merendi G.A. \& Vinci A. (1994) - Sistemi di Paesaggio della Toscana, Giunta Regionale della Toscana, Firenze, $157 \mathrm{pp}$.

Sestini A. (1963) - Il Paesaggio, Volume 7: Conosci l'Italia, Touring Club Italiano, Milano, $232 \mathrm{pp}$.

Trevisani M. \& Sassoli U. (2014) - L'informazione geografica nella Regione Toscana. Ri-Vista 1-2/2014, Firenze, 138145 . 